\title{
LETTERS
}

\section{NLRX1 is a regulator of mitochondrial antiviral immunity}

\author{
Chris B. Moore ${ }^{1,2}$, Daniel T. Bergstralh ${ }^{2}$, Joseph A. Duncan ${ }^{3}$, Yu Lei $^{1,2}$, Thomas E. Morrison ${ }^{4,5}$, \\ Albert G. Zimmermann ${ }^{1,2}$, Mary A. Accavitti-Loper ${ }^{7}$, Victoria J. Madden ${ }^{6}$, Lijun Sun ${ }^{8}$, Zhengmao Ye ${ }^{1,2}$, \\ John D. Lich ${ }^{1,2}$, Mark T. Heise ${ }^{4,5}$, Zhijian Chen ${ }^{8} \&$ Jenny P-Y. Ting ${ }^{1,2}$
}

The RIG-like helicase (RLH) family of intracellular receptors detect viral nucleic acid and signal through the mitochondrial antiviral signalling adaptor MAVS (also known as Cardif, VISA and IPS-1) during a viral infection ${ }^{1-6}$. MAVS activation leads to the rapid production of antiviral cytokines, including type 1 interferons. Although MAVS is vital to antiviral immunity, its regulation from within the mitochondria remains unknown. Here we describe human NLRX1, a highly conserved nucleotide-binding domain (NBD)- and leucine-rich-repeat (LRR)-containing family member (known as NLR) that localizes to the mitochondrial outer membrane and interacts with MAVS. Expression of NLRX1 results in the potent inhibition of RLH- and MAVS-mediated interferon- $\beta$ promoter activity and in the disruption of virus-induced RLHMAVS interactions. Depletion of NLRX1 with small interference RNA promotes virus-induced type $I$ interferon production and decreases viral replication. This work identifies NLRX1 as a check against mitochondrial antiviral responses and represents an intersection of three ancient cellular processes: NLR signalling, intracellular virus detection and the use of mitochondria as a platform for anti-pathogen signalling. This represents a conceptual advance, in that NLRX1 is a modulator of pathogen-associated molecular pattern receptors rather than a receptor, and identifies a key therapeutic target for enhancing antiviral responses.

Mammalian members of the nucleotide-binding domain (NBD) and leucine-rich-repeat-containing (LRR) (known as NLR, see http://www.genenames.org/genefamily/nacht.html) family of proteins are indispensable for cellular responses to pathogens. This NBD-LRR protein structure is ancient and highly conserved, as shown by its initial identification among plant disease-resistance proteins ${ }^{7-12}$. Current dogma posits that NLRs function as cytoplasmic surveillance molecules that sense intracellular pathogen-associated molecular patterns (PAMPs), or as regulators of pathogen-initiated signalling cascades ${ }^{13,14}$. Viral PAMPs are detected by the cytoplasmic RLH receptors RIG-I (also known as DDX58) and MDA-5 (also known as IFIH1), which signal through the mitochondrial protein MAVS, resulting in the activation of interferon regulatory factor 3 (IRF3) and NF- $\kappa B$ and type-1 interferon transcription ${ }^{1-6}$. Abrogation of MAVS expression or function leads to reduced type 1 interferon production and antiviral protection ${ }^{15}$.

To study the potential role of NLR proteins in regulating mitochondrial antiviral signalling, we used bioinformatics to identify NLRs localized to the mitochondria. We identified one putative mitochondrial NLR called NLRX1 (previously known as CLR11.3 and NOD9 $)^{9,16}$ (Fig. 1a). The predicted peptide sequence and distinct domains of NLRX1 are shown in Supplementary Fig. 1. Consistent with the conserved motif structure of the NLR family, NLRX1 contains a central putative NBD and carboxy-terminal LRRs. The assignment of the amino-terminal effector domain to a subclass is less clear. Instead, the $\mathrm{N}$ terminus has evolved to include a mitochondrialtargeting sequence (Supplementary Fig. 1). On the basis of hydrophobicity analyses, we identified two putative transmembrane regions (Supplementary Fig. 2). NLRX1 homologues were identified in all vertebrates examined, with a remarkably high (92.4\%) degree of conservation between human and mouse (Supplementary Fig. 3).

To investigate NLRX1 function, NLRX1 was isolated and the complementary DNAs encoding $\mathrm{N}$ and $\mathrm{C}$ termini were verified by rapid amplification of cDNA ends (RACE). The 3' RACE produced two products, identifying a splice variant lacking a portion of the LRR region encoded by exon 9 (Supplementary Fig. 4). NLRX1 messenger RNA is broadly expressed, suggesting a ubiquitous role (Supplementary Fig. 5). To examine protein expression, we generated an antiNLRX1 monoclonal antibody. Antibody specificity was verified by immunoblotting against transfected, epitope-tagged haemagglutinin (HA)-NLRX1 or another NLR protein HA-NLRP12 (also known as Monarch1; ref. 17). NLRX1 antibody detected a strong band corresponding to the exogenous HA-tagged NLRX1 (Supplementary Fig. 6) and a weaker endogenous product of similar size in cells transfected with pcDNA or with HA-tagged NLRP12 (Supplementary Fig. 6). Short interfering RNA (siRNA)-mediated knockdown of NLRX1 greatly reduced the detection of this endogenous product, whereas control siRNA oligonucleotides had no effect (Supplementary Fig. 7). NLRX1 protein was also detected in all cell lines analysed, further suggesting ubiquitous expression (Supplementary Fig. 8).

Additional clues to NLRX1 function are revealed by its cellular localization. Both endogenous and overexpressed NLRX1 revealed a punctate cytoplasmic distribution (Fig. 1b and Supplementary Fig. 9a). Biochemical fractionation verified that endogenous NLRX1 protein resided in the non-nuclear fraction (Fig. 1c, middle panel). This fraction contains both cytosol and cytoplasmic organelles, the latter of which include the mitochondria. Separation of this cytoplasmic/ organellar fraction into three fractions (small membranes, soluble cytosol and mitochondria) revealed that NLRX1 was found exclusively in the mitochondrial fraction, which also contained the mitochondrial protein TOM20 (translocase of outer mitochondrial membrane 20 homologue; also known as TOMM20) (Fig. 1c, right panel). A merged confocal image of endogenous NLRX1 and the mitochondrial stain Mitotracker indicated mitochondrial localization of NLRX1 (Supplementary Fig. 9b). Antibody staining by immunogold-bead electron microscopy was used as the ultimate method for visualizing NLRX1; this localized NLRX1 to the 
mitochondria with little staining elsewhere (Fig. 1d, top panels). Costaining for NLRX1 (small beads) and MAVS (large beads) identified several mitochondria containing both proteins (Fig. 1d, middle panels). Isotype control antibody showed no nonspecific staining (Fig. 1d, bottom left panel), whereas the positive control TOM20 showed mitochondrial staining (Fig. 1d, bottom right panel). As observed for MAVS and TOM20, many of the NLRX1-specific immunogold beads were proximal to the mitochondrial membrane. To study this further, we separated mitochondrial membrane fractions by a sucrose gradient (Fig. 1e). Voltage-dependent anion channel 1 (VDAC1) and MAVS are proteins known to reside in the mitochondrial outer membrane and served as positive controls ${ }^{1,18}$. VDAC1, MAVS and NLRX1 were the only proteins detected in fractions 1-5, which includes primarily mitochondrial outer membrane proteins (Fig. 1e). COXIV (cytochrome c oxidase subunit IV isoform 1; also known as COX4I1) is an inner membrane mitochondrial protein and was only found in fractions 6-10. To confirm this finding, a trypsin protection assay was performed on prepared mitochondrial fractions.
NLRX1 and the known outer membrane proteins MAVS and BCL2L1 were all sensitive to proteolytic cleavage by trypsin, whereas the mitochondrial inner membrane protein COXIV was completely protected (Fig. 1f). Furthermore, deletion of the N-terminal mitochondrial targeting sequence resulted in a loss of mitochondrial localization (Supplementary Fig. 10). These results indicate that NLRX1 resides in the outer mitochondrial membrane.We next examined the interaction of NLRX1 and MAVS. Co-immunoprecipitation studies demonstrate that HA-NLRX1 interacts with MAVS (Fig. 2a) but not with other known mitochondrial outer membrane proteins (BCL2 and BCL2L1), indicating specificity of the NLRX1-MAVS interaction (Fig. 2b). Finally, endogenous NLRX1 associates strongly with endogenous MAVS after immunoprecipitation with two different MAVS antibodies (Fig. 2c). Consistent with these results, MAVS and NLRX1 show a remarkably similar expression level in many cell types (Supplementary Fig. 11).

NLRX1 and MAVS are both modular proteins; therefore, we sought to investigate the specific domains required for this interaction. The

\begin{tabular}{lccc}
\hline a & & & \\
\hline NLRX1 (CLR11.3/NOD9) & Mitochondria & Cytosol & Prediction \\
NLRP12 (Monarch1/CLR19.3/PYPAF7/NALP12/PAN6) & $52 \%$ & $22 \%$ & Mito. \\
NLRP2 (CLR19.9/NBS1/PYPAF2/NALP2) & $26 \%$ & $35 \%$ & Cytosol \\
NLRP3 (CIAS1/NALP3/Pypaf1) & $17 \%$ & $48 \%$ & Cytosol \\
NLRP4 (CLR19.5/RNH2/PYPAF4/NALP4/PAN2) & $17 \%$ & $39 \%$ & Cytosol \\
NLRP6 (CLR11.4/PAN3/PYPAF5/NALP6) & $17 \%$ & $35 \%$ & Cytosol \\
NLRP13 (CLR19.7/NOD14/PAN13/NALP13) & $17 \%$ & $35 \%$ & Cytosol \\
NAIP (BIRC1/CLR5.1/NLRB1) & $17 \%$ & $48 \%$ & Cytosol \\
NLRP14 (CLR11.2/NOD5/NALP14/PAN8) & $17 \%$ & $52 \%$ & Cytosol \\
CIITA (NLRA/MHCC2TA) & $13 \%$ & $39 \%$ & Cytosol \\
NLRP9 (CLR19.1/NOD6/NALP9/PAN12) & $13 \%$ & $26 \%$ & Nuclear \\
NOD2 (CARD15/CLR16.3/NLRC2) & $9 \%$ & $91 \%$ & Cytosol \\
NLRP7 (CLR19.4/Nod12/NALP7/PAN7) & $9 \%$ & $52 \%$ & Cytosol \\
NLRP10 (CLR11.1/NOD8/NALP10/PAN5/PYNOD) & $4 \%$ & $52 \%$ & Cytosol \\
NLRC3 (CLR16.2/NOD3) & $4 \%$ & $61 \%$ & Cytosol \\
NLRC4 (IPAF/CARD12/CLR2.1) & $4 \%$ & $48 \%$ & Cytosol \\
NRRA & $0 \%$ & $52 \%$ & Cytosol \\
\hline
\end{tabular}

b

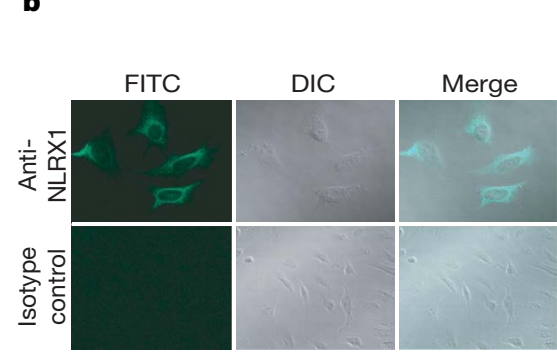

c

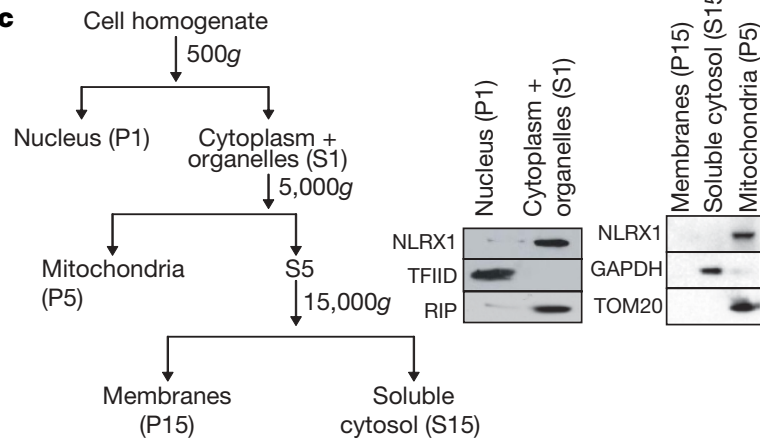

d

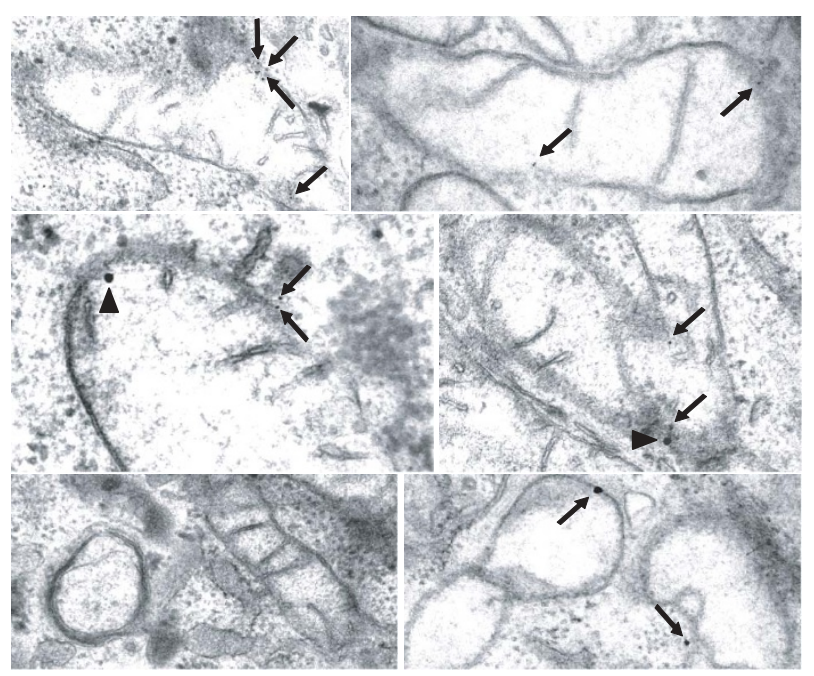

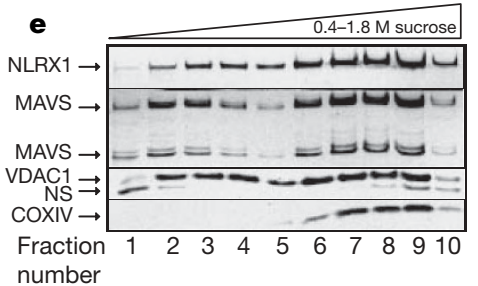

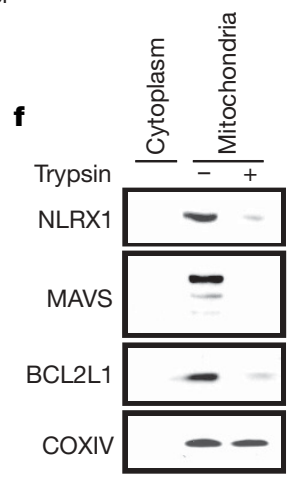

Figure 1 | NLRX1 is an outer membrane mitochondrial protein. a, Prediction of subcellular localizations for NLRs. This was calculated using K-nearest neighbours (k-NN) classifier predictions of representative NLR localizations. Listed are the official gene symbols followed by aliases in parentheses. b, Cytoplasmic localization of endogenous NLRX1. FITC, fluorescein isothiocyanate; DIC, differential interference contrast. c, Left, fractionation scheme used. Right, immunoblot of NLRX1 in various cell fractions. d, Immunogold-bead electron micrographs of NLRX1 alone (top panels), NLRX1 (small bead, arrow) and MAVS (large bead, arrowhead) (middle panels), isotype antibody control (bottom left panel), and TOM20 (bottom right panel). e, Immunoblotting of NLRX1 and known outer (VDAC1 and MAVS) and inner (COXIV) mitochondrial proteins in membrane fractions. NS denotes nonspecific protein band. f, Trypsin treatments of mitochondria followed by immunoblotting with NLRX1, MAVS and BCL2L1(outer membrane), as well as COXIV (inner membrane). 
a

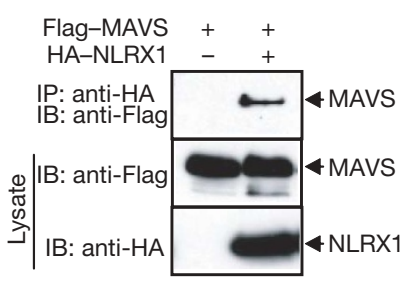

d

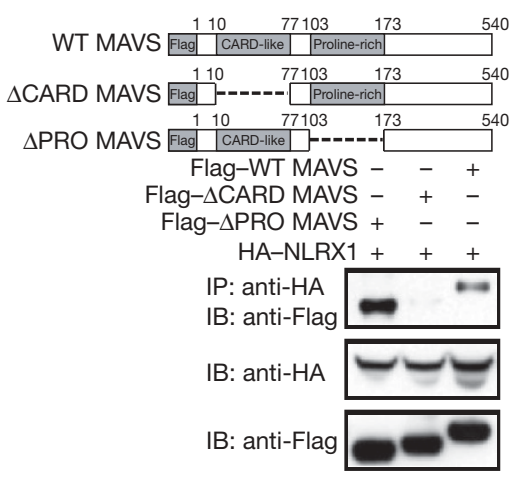

b

Flag-BCL2L1 - - + Flag-MAVS - + - -

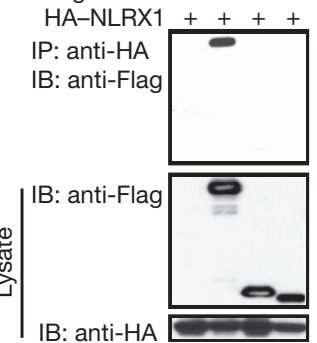

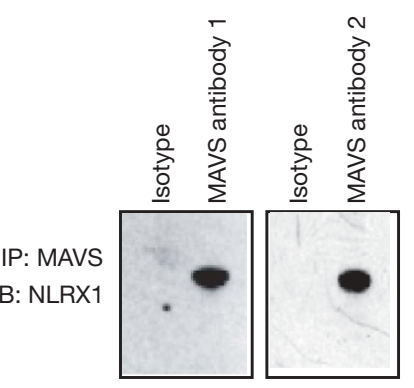

Figure 2 | NLRX1 interacts with MAVS. a, Interaction of overexpressed NLRX1 and MAVS. IP and IB denote immunoprecipitation and immunoblotting, respectively. b, Interactions of NLRX1 with other known mitochondrial outer membrane proteins. c, Interaction of endogenous NLRX1 with endogenous MAVS. d, Interaction of MAVS truncation mutants with wild-type (WT) NLRX1. e, Interaction of NLRX1 truncation mutants with wild-type MAVS. Deletion constructs are shown above; $15 \%$ denotes $15 \%$ polyacrylamide gel necessary to resolve the smallest protein. The $\mathrm{X}$ domain is currently undefined.

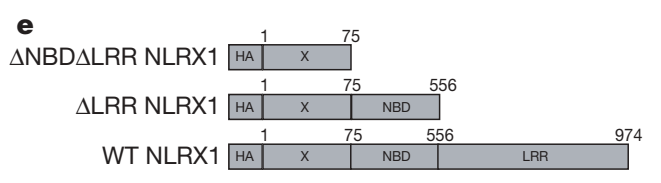

WT NLRX1

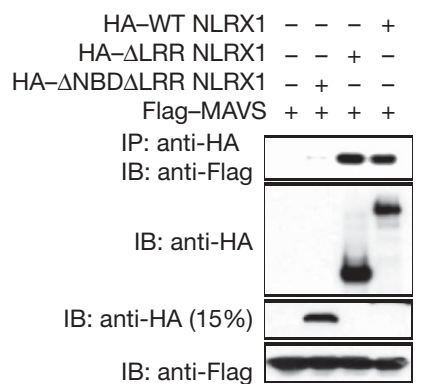

$\mathrm{N}$-terminal CARD domain of MAVS is required for MAVS signalling and interactions with RLH family members ${ }^{1}$. It is also required for the interaction with NLRX1 (Fig. 2d). In NLRX1, deletion of the LRR had no effect on the NLRX1-MAVS interaction. A further deletion of the putative NBD disrupted this interaction, indicating that this domain is required for maintaining the interaction with MAVS (Fig. 2e). Collectively, these data demonstrate that NLRX1 is a MAVS-associated mitochondrial outer membrane protein and raise the intriguing possibility that it participates in antiviral signalling.

Signalling in response to the synthetic viral double-stranded RNA (dsRNA) analogue poly(I:C) is mediated both at the cell membrane through Toll-like receptor 3 (TLR3) and within the cytoplasm by means of direct binding to the RLH molecules to activate MAVS. Both pathways activate NF- $\kappa \mathrm{B}$ and IRF3, leading to interferon $\beta$ (IFN- $\beta$ ) transcription ${ }^{5}$. Poly(I:C), cannot activate IFN- $\beta$ expression in TLR3-deficient 293T cells when applied extracellularly. However, when delivered into the cytoplasm by transfection, poly(I:C) stimulates IFN- $\beta$ through the MAVS pathway. Conversely, stable TLR3expressing 293T cells have a robust response to extracellular poly(I:C), which does not require MAVS signalling. We used these two cell lines in poly(I:C)-induced IFN- $\beta$ luciferase experiments to delineate a role for NLRX1 in intracellular (RLH) versus extracellular (TLR) antiviral responses. NLRX1 had no effect on the TLR3mediated extracellular activation of IFN- $\beta$ or NF- $\kappa B$ luciferase repor-
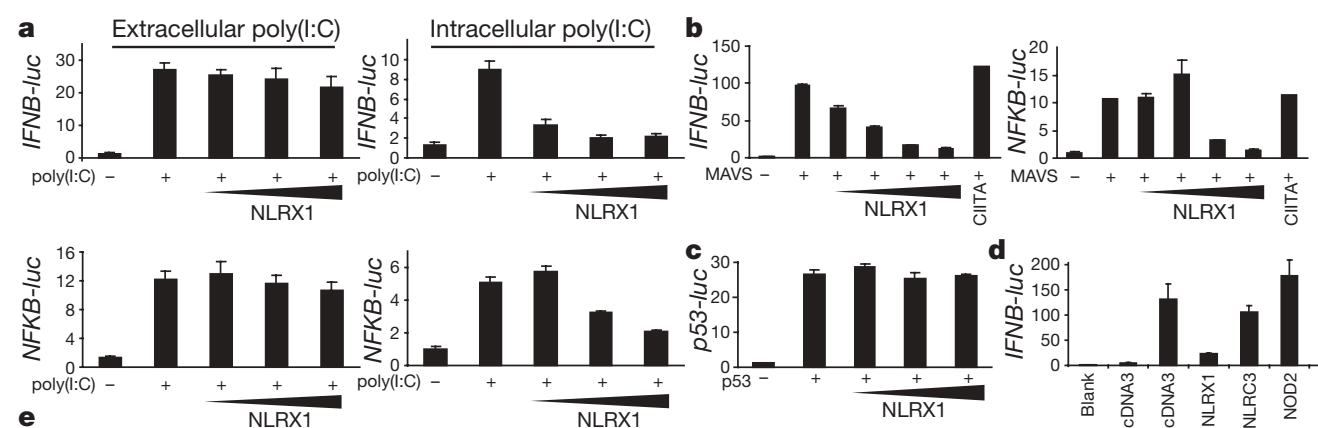

IIII
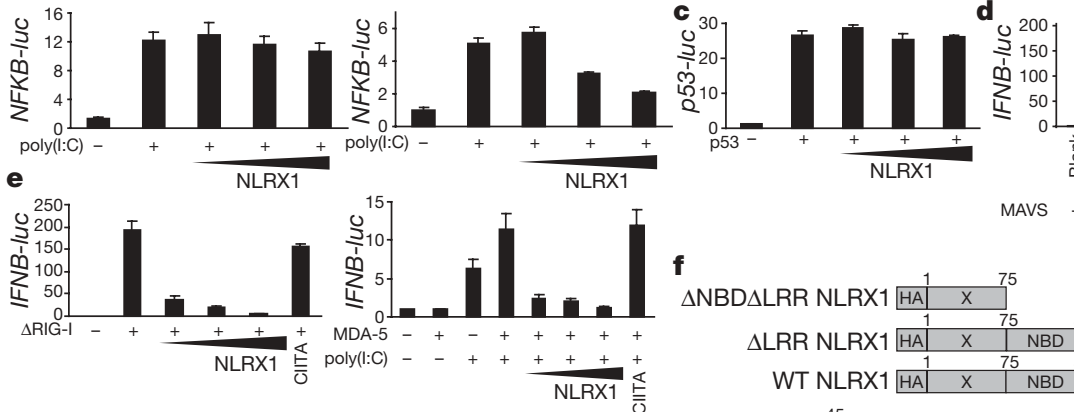

f
Figure 3 | NLRX1 inhibits MAVSmediated activation of IFN- $\beta$ and NF-кB. a-c, Effect of NLRX1 on IFNB- and NFKB-luciferase (luc) activation by extracellular and intracellular poly(I:C) (a); IFNB-luc (left) and NFKB-luc (right) activation by MAVS (b); and p53 activity (c). d, IFNB-luc is inhibited by NLRX1 but not by other NLR proteins. e, Effect of NLRX1 on $\triangle$ RIG-I- (left) and MDA-5- (right) mediated activation of IFNB-luc activity. $\mathbf{f}$, Repression of MAVSmediated IFNB-luc by wild-type (WT) NLRX1 compared with its truncation mutants. Data from a-f are presented as mean \pm s.d. from three independent experiments. 
ters (Fig. 3a, top and bottom left panels). By contrast, the intracellular IFN- $\beta$ response was strongly inhibited by NLRX1 (Fig. 3a, top right panel). NF- $\kappa \mathrm{B}$ luciferase reporter activation was likewise reduced, although to a lesser extent (Fig. 3a, bottom right panel). Thus, NLRX1 acts as a negative regulator within the intracellular antiviral pathway.

We next sought to study MAVS activation of IFN- $\beta$ directly. MAVS potently activated the IFN- $\beta$ reporter luciferase, and NLRX1 inhibited this activation in a dose-dependent fashion (Fig. 3b, left). MAVS-induced NF- $\kappa B$ luciferase activity was inhibited by NLRX1, but to a lesser extent (Fig. 3b, right). As a specificity control, p53 luciferase reporter was not affected by NLRX1 (Fig. 3c). Other NLR proteins (CIITA, NOD2 and NLRC3 (also known as CLR16.2)) did not significantly affect IFN- $\beta$ luciferase (Fig. 3d). Expression of the constitutively active truncation mutant $\triangle R I G-I$ induces a robust intracellular antiviral response through MAVS $^{19,20}$. NLRX1 significantly reduced the ability of $\Delta$ RIG-I to activate IFN- $\beta$ transcription (Fig. 3e, left). MDA-5 is the intracellular receptor for poly(I:C) and picornavirus nucleic acid and also requires MAVS $^{6}$. NLRX1 also abolished MDA-5 activity (Fig. 3e, right). Furthermore, mapping of the NLRX1 functional domain revealed that the C-terminal LRR domain is required for the repression of MAVS-induced interferon signalling (Fig. 3f). These data indicate that NLRX1 functions as an inhibitor of RLH-mediated MAVS antiviral signalling through the NLRX1 LRR domain.

To confirm that endogenous NLRX1 also repressed IFN- $\beta$ production, NLRX1-specific siRNA oligonucleotides, which reduced NLRX1 protein by greater than $90 \%$ (Supplementary Fig. 7), were used. As expected, IFNB and IFNA mRNA levels rose sharply by $16 \mathrm{~h}$ post-transfection with MAVS. Both interferons were significantly increased in cells containing NLRX1 siRNA (Fig. 4a). This increased MAVS-mediated response was also observed with the NF- $\kappa \mathrm{B}-$ responsive genes interleukin 6 (IL6), RANTES (also known as CCL5) and tumour necrosis factor $\alpha(T N F a)$, although to a lesser extent, consistent with the aforementioned NF- $\kappa \mathrm{B}$ luciferase data (Fig. 4a). To test the effect of NLRX1 during a viral infection, IFNB mRNA and protein levels were quantified in NLRX1-deficient cells infected with Sendai virus, which activates RIG-I and MAVS for type1 interferon production ${ }^{1}$. IFNB mRNA induction by Sendai virus was increased $>8$-fold in cells with NLRX1 siRNA compared with control siRNA (Fig. 4b, left). Consistent with the transcriptional data, the Sendai-virus-induced IFN- $\beta$ protein level was also greater in the infected NLRX1 siRNA cells (Fig. 4b, right). In addition, NLRX1 expression attenuated MAVS, $\triangle R I G-I$ and Sendai-virus-induced IRF3 dimer formation, indicating that NLRX1 reduced MAVSmediated IRF3 signalling (Fig. 4c).

To identify a mechanism for the inhibitory effect of NLRX1 on antiviral signalling, we examined the effect of NLRX1 on the endogenous association of MAVS with RIG-I. The interaction of MAVS and RIG-I was enhanced by Sendai viral infection, whereas the introduction of NLRX1 eliminated this enhancement (Fig. 4d). Because this RLH-MAVS interaction is required for signalling, the disruption of this interaction by NLRX1 should squelch further downstream antiviral signalling. We extended these results by measuring cell death, viral replication and IFN- $\beta$ production in NLRX1 siRNA cells infected with the human alphavirus Sindbis. In congruence with the Sendai-virus findings, NLRX1 siRNA cells infected with Sindbis produced more IFN- $\beta$ protein than control siRNA cells (Fig. 4e, left) and the cells were highly resistant to green fluorescent protein (GFP)Sindbis viral replication $(25.06 \%)$ compared with the control siRNA cells $(83.87 \%)$ (Fig. 4e, right). The differences in cell death are not significant and therefore can not account for the large difference in viral susceptibility (Supplementary Fig. 12). Cumulatively, these observations solidify the negative regulatory role of NLRX1 in MAVS-mediated antiviral responses, and demonstrate that it is mediated through the inhibition of virus-induced RLH-MAVS interactions.
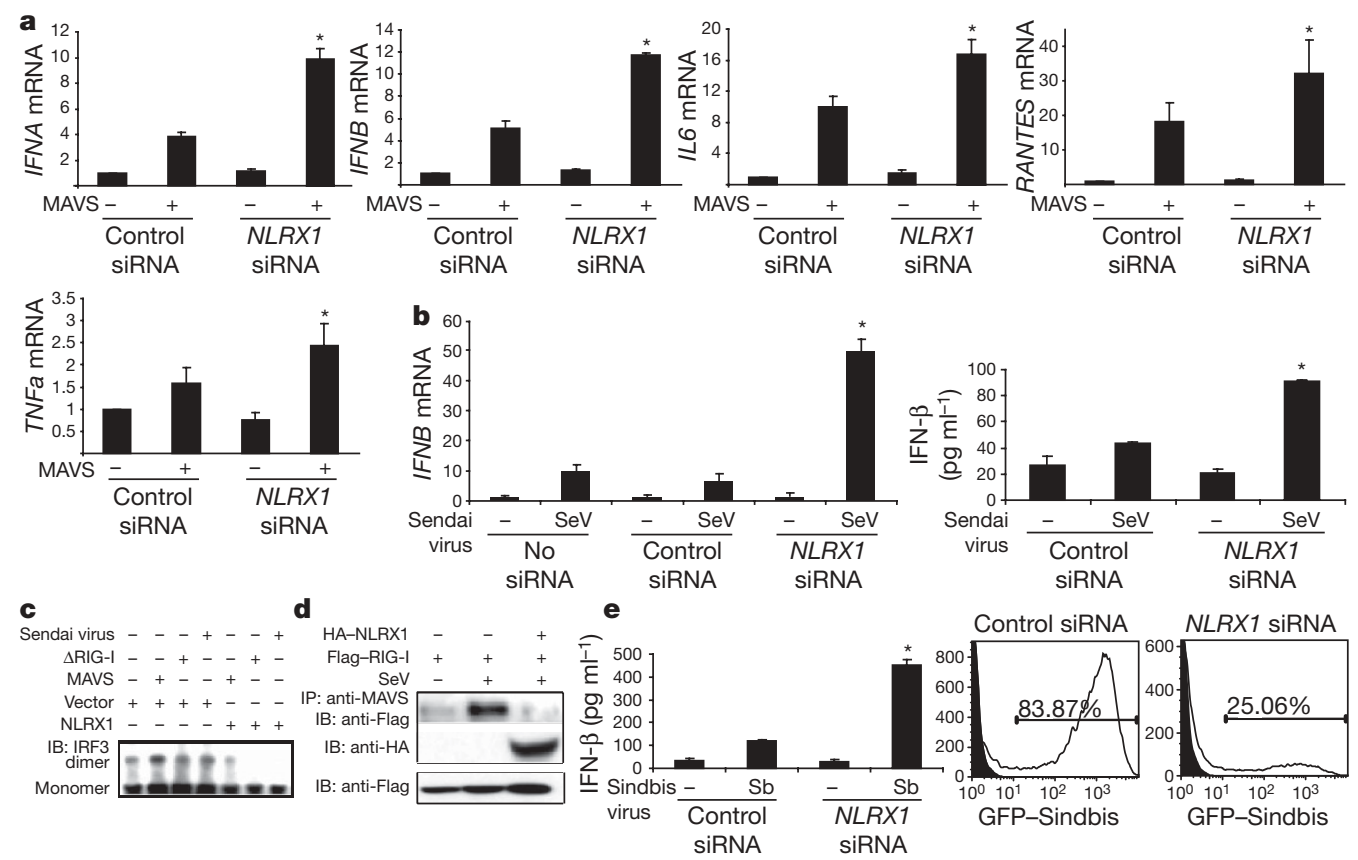

Figure 4 | NLRX1 siRNA results in enhanced antiviral responses. a, Activation of IFNB, IFNA and the NF- $\kappa \mathrm{B}$ responsive genes IL6, RANTES and TNFa by MAVS in the presence of control or NLRX1-targeted siRNA as measured by quantitative real-time PCR. All results were normalized to $18 \mathrm{~S}$ rRNA values. b, IFNB mRNA (left) and protein (right) in Sendai-virus $(\mathrm{SeV})$-infected HEK293T cells in the presence of control non-targeting or NLRX1-targeted siRNA. Data from $\mathbf{a}$ and $\mathbf{b}$ are presented as means \pm s.d. from three independent experiments. c, Immunoblotting of IRF3 in native whole-cell lysates from MAVS, $\triangle$ RIG-I and Sendai-virus-infected cells after overexpression of empty vector or NLRX1. d, Co-immunoprecipitation of endogenous MAVS with overexpressed RIG-I following mock or Sendai virus infections in the presence or absence of overexpressed NLRX1. e, Left panel, IFN- $\beta$ protein levels from Sindbis-virus-infected $293 \mathrm{~T}$ cells

containing control or NLRX1 siRNA. Data are presented as means \pm s.d. of triplicate determinations from two independent experiments. Right panel, percentage of GFP-Sindbis-positive cells in 293T cells containing control or NLRX1 siRNA as analysed by flow cytometry. 
Interestingly, NLRX1 mRNA and protein levels are stable after activation of MAVS signalling (Supplementary Fig. 13); therefore, the regulation of NLRX1 apparently does not involve simple transcriptional or translational mechanisms. This report shows a linkage between the rapidly emerging NLR family and mitochondrial antiviral signalling. We speculate that the mitochondrial membrane provides a convenient surface on which antiviral cell-signalling complexes are arranged and activated. Rather than directly engaging a pathogen-derived product, NLRX1 seems to function as a modulator of PAMP receptors (MOPR) by affecting the interaction of the pathogen receptor RIG-I (and possibly MDA-5) with MAVS. This mechanism is more in-line with the Guard hypothesis proposed for the indirect detection of pathogen products by plant $\mathrm{R}$ proteins ${ }^{21,22}$. Because both NLRX1 and RIG-I interact with the CARD domain of MAVS, a probable scenario is that NLRX1 and RIG-I compete for binding with the CARD domain of MAVS, with opposing outcomes. Because MAVS is a potent activator of type 1 interferon, a brake within this mitochondrial signalling complex could prevent unwarranted deleterious antiviral responses and regulate interferon signalling during the course of a viral infection. This report shows that NLRX1 is such a brake. It further demonstrates that targeting of NLRX1 through approaches such as siRNA could enhance antiviral responses, which has broad implications for the treatment of viralassociated diseases.

\section{METHODS SUMMARY}

Cloning of NLRX1. A complete open reading frame of human NLRX1 was isolated from Jurkat cDNA using standard PCR techniques. The complete N and $\mathrm{C}$ terminals were verified by RACE.

Expression data and antibody production. Expression data for NLRX1 were mined from the Genomics Institute of the Novartis Research Foundation. Recombinant hexahistidine-tagged NLRX1 was used as an antigen for the production of monoclonal antibody. NLRX1 antigen was injected into mice and monoclonal antibodies were produced by standard methodology.

Real-time PCR. First-strand cDNA was generated from total RNA using random priming and moloney murine leukemia virus (MMLV) reverse transcriptase (Invitrogen). Real-time PCR was performed using QuantiTect SYBR Green PCR Master Mix (Qiagen) in triplicate experiments and analysed on an $A B$ Prism 7700 analyser (Applied Biosystems). All real-time values were normalized to $18 \mathrm{~S}$ ribosomal RNA.

Immunofluorescent staining, immunogold and mitochondrial localizations. HeLa cells were transfected with $1.0 \mu \mathrm{g}$ per well GFP-NLRX1 or stained with NLRX1 antibody. Anti-mouse Alexa Fluor 488 (Molecular Probes) was used to stain for endogenous NLRX1. All exogenous and endogenous NLRX1 localizations were visualized on a Zeiss LSM5 PASCAL confocal microscope. To determine co-localization with mitochondria, HeLa cells were co-stained with Mitotracker (Invitrogen) and endogenous NLRX1. Transmission electron microscopy of anti-NLRX1 immunogold labelling was also performed by standard methods. NLRX1 protein was detected in sucrose gradient fractions and trypsin-treated mitochondrial fractions.

Luciferase assays, co-immunoprecipitations and siRNA. HEK293T or TLR3HEK293T cells were transfected in 96-well plates with IFN- $\beta$, NF- $\kappa B$ or p53 luciferase constructs and HA-NLRX1. Exogenous MAVS, poly(I:C) or p53 plasmid was used as an agonist. All co-immunoprecipitation studies of exogenous and endogenous proteins were performed from whole cells lysed in RIPA buffer and following standard methodology. Non-targeting, glyceraldehyde 3-phosphate dehydrogenase $(G A P D H)$ or NLRX1 siRNA oligonucleotides (Dharmacon) were transfected following the commercial protocol.

Full Methods and any associated references are available in the online version of the paper at www.nature.com/nature.

Received 14 November; accepted 22 November 2007. Published online 16 January 2008.

1. Seth, R. B., Sun, L., Ea, C. K. \& Chen, Z. J. Identification and characterization of MAVS, a mitochondrial antiviral signaling protein that activates NF- $\mathrm{KB}$ and IRF 3. Cell 122, 669-682 (2005)
2. Meylan, E. et al. Cardif is an adaptor protein in the RIG-I antiviral pathway and is targeted by hepatitis C virus. Nature 437, 1167-1172 (2005).

3. Kawai, T. et al. IPS-1, an adaptor triggering RIG-I- and Mda5-mediated type I interferon induction. Nature Immunol. 6, 981-988 (2005).

4. $\mathrm{Xu}, \mathrm{L}$. G. et al. VISA is an adapter protein required for virus-triggered IFN- $\beta$ signaling. Mol. Cell 19, 727-740 (2005).

5. Meylan, E. \& Tschopp, J. Toll-like receptors and RNA helicases: two parallel ways to trigger antiviral responses. Mol. Cell 22, 561-569 (2006).

6. Gitlin, L. et al. Essential role of mda-5 in type I IFN responses to polyriboinosinic:polyribocytidylic acid and encephalomyocarditis picornavirus. Proc. Natl Acad. Sci. USA 103, 8459-8464 (2006).

7. Harton, J. A., Linhoff, M. W., Zhang, J. \& Ting, J. P. Cutting edge: CATERPILLER: a large family of mammalian genes containing CARD, pyrin, nucleotide-binding, and leucine-rich repeat domains. J. Immunol. 169, 4088-4093 (2002).

8. Inohara, N. \& Nunez, G. NODs: intracellular proteins involved in inflammation and apoptosis. Nature Rev. Immunol. 3, 371-382 (2003).

9. Inohara, N. Chamaillard, M. McDonald, C. \& Nunez, G. NOD-LRR proteins: role in host-microbial interactions and inflammatory disease. Annu. Rev. Biochem. 74, 355-383 (2005)

10. Martinon, F. \& Tschopp, J. NLRs join TLRs as innate sensors of pathogens. Trends Immunol. 26, 447-454 (2005).

11. Bruey, J. M. et al. PAN1/NALP2/PYPAF2, an inducible inflammatory mediator that regulates NF-KB and caspase-1 activation in macrophages. J. Biol. Chem. 279, 51897-51907 (2004).

12. Jones, J. D. \& Dangl, J. L. The plant immune system. Nature 444, 323-329 (2006).

13. Girardin, S. E. et al. Nod1 detects a unique muropeptide from Gram-negative bacterial peptidoglycan. Science 300, 1584-1587 (2003).

14. Inohara, N. et al. Host recognition of bacterial muramyl dipeptide mediated through NOD2. Implications for Crohn's disease. J. Biol. Chem. 278, 5509-5512 (2003).

15. Sun, Q. et al. The specific and essential role of MAVS in antiviral innate immune responses. Immunity 24, 633-642 (2006).

16. Ting, J. P. \& Davis, B. K. CATERPILLER: a novel gene family important in immunity, cell death, and diseases. Annu. Rev. Immunol. 23, 387-414 (2005).

17. Williams, K. L. et al. The CATERPILLER protein monarch-1 is an antagonist of Tolllike receptor-, tumor necrosis factor $\alpha$-, and Mycobacterium tuberculosis-induced pro-inflammatory signals. J. Biol. Chem. 280, 39914-39924 (2005).

18. Shimizu, S., Narita, M. \& Tsujimoto, Y. Bcl-2 family proteins regulate the release of apoptogenic cytochrome $\mathrm{c}$ by the mitochondrial channel VDAC. Nature 399, 483-487 (1999)

19. Sharma, S. et al. Triggering the interferon antiviral response through an IKKrelated pathway. Science 300, 1148-1151 (2003).

20. Yoneyama, M. et al. The RNA helicase RIG-I has an essential function in doublestranded RNA-induced innate antiviral responses. Nature Immunol. 5, 730-737 (2004).

21. Marathe, R. \& Dinesh-Kumar, S. P. Plant defense: one post, multiple guards?! Mol. Cell 11, 284-286 (2003).

22. DeYoung, B. J. \& Innes, R. W. Plant NBS-LRR proteins in pathogen sensing and host defense. Nature Immunol. 7, 1243-1249 (2006)

Supplementary Information is linked to the online version of the paper at www.nature.com/nature.

Acknowledgements This work was supported by National Institute of Health SERCEB (J.P.-Y.T.). J.P.-Y.T is a Sandlers Program Awardee. This work was supported by a Pfizer Fellowship in Infectious Disease (J.A.D) as well as a National Institute of Health Institutional National Research Service Award Postdoctoral Training Fellowship and a Juvenile Diabetes Research Foundation International Postdoctoral Training Fellowship (C.B.M.). We thank R. Bagnell Jr, B. Conti, B. Davis and W. O'Connor for technical assistance. We also thank G. Cheng and E. Pietras for discussions.

Author Contributions C.B.M. and J.P.-Y.T. designed the experiments and prepared the manuscript. C.B.M. performed the experiments. D.T.B. performed several experiments and assisted with the manuscript. J.A.D. performed cell fractionation studies and produced antigen for antibody production. Y.L. tested antibody specificity and provided considerable technical assistance. M.T.H. and T.E.M. assisted with all viral studies. A.G.Z. created NLRX1 truncation mutants. M.A.A-L. produced monoclonal antibody and provided several hybridomas for analysis. V.J.M. performed immunogold-bead transmission electron microscopy. L.S. performed mitochondrial fractionation by sucrose gradient. Z.Y. performed flow cytometry, and J.D.L. assisted with co-immunoprecipitation studies.

Author Information Reprints and permissions information is available at www.nature.com/reprints. Correspondence and requests for materials should be addressed to J.P.-Y.T. (jenny_ting@med.unc.edu). 


\section{METHODS}

Cell culture. HeLa, HEK293T and TLR3-HEK293T cells were maintained in DMEM-H medium supplemented with $10 \%$ fetal calf serum and grown in a humidified $37^{\circ} \mathrm{C}$ incubator with $5 \% \mathrm{CO}_{2}$. TLR3-HEK293T cells (Invivogen) were grown under selection in DMEM-H and $10 \mu \mathrm{g} \mathrm{ml}^{-1}$ blasticidin (Sigma). Cloning of human NLRX1 and truncation mutants. Jurkat total mRNA was isolated using the RNeasy kit (Qiagen) and cDNA generated with Transcriptor Reverse Transcriptase (Roche Applied Science). PCRs were performed with Pfx DNA polymerase (Invitrogen). The following primers were used to generate a complete open reading frame of human NLRX1: forward, 5'-ATACGCCACCACGGAAG; reverse, 5'-TGGCCTGGGCATTCTC. RACE was performed using the RLM-RACE kit (Ambion) and the following oligonucleotides: 5'RACE, 5'-AGGCAGCCACAGACATCCTC (pos1313), 5'-TCCTTCGTCCGTCGCCGATA (pos1065) and 5'-TGATGCTCCAGGGCCAGCTC (pos359); 3'RACE, 5'-ATGTTTCGAGAGAGGAGGACTAC (pos1699). RACE fragments were cloned and full-length NLRX1 assembled by splice overlap extension. HA- and GFP-tagged NLRX1 plasmids were constructed by cloning into HA-pcDNA3 (Invitrogen) and pEGFP-C3 (Clontech), respectively. A BLAST query of human NLRX1 against all known genomes was performed, and multiple sequence alignments of all identified NLRX1 homologues were assembled using Vector NTI (Invitrogen). The human $\triangle$ LRR NLRX1 mutant was created by performing PCR with Takara LA Taq (Takara Bio, Inc.) using the forward primer 5'-AGCTTGGTACCGAGGTGGGGCCACCATTTGCCCAGGGCC-3' (primer 1) and the reverse primer $5^{\prime}$-ATAACGGTACCTAATAGATGCTTGTAAGGGTCTGCCCAGC- $3^{\prime}$. The $\triangle$ NBD $\triangle$ LRR NLRX1 truncation mutant was created using the forward primer 1 and the reverse primer $5^{\prime}$-ATAACGGTACCTAAGTTGCAGAGGCGCTGGGGAACAG-3' (primer 2). The $\triangle$ MTS NLRX1 was created using the forward primer 5'-AGCTTGGTACCGGAAGCTATACAGCGGCACCGCCGGAAC-3' and reverse primer 2. All MAVS truncation mutants were created as described previously ${ }^{1}$.

Expression data and antibody production. Cell and tissue expression of NLRX1 was obtained from the Genomics Institute of the Novartis Research Foundation (symatlas.gnf.org/SymAtlas) using NOD9 as the keyword identifier ${ }^{23}$. Subcellular localizations and transmembrane regions were predicted using PSORTII and TmPred software, respectively ${ }^{24}$. Recombinant hexahistidinetagged NLRX1 (amino acids 1 to 463) was used as the antigen for production of monoclonal antibody. The indicated amino acids were cloned into the bacterial expression vector PQE-80LIC using the PCR- and ligation-independent cloning as described previously ${ }^{25}$. The recombinant protein fragment was expressed in E. coli strain BL21codon plus (Stratagene) and purified from inclusion bodies by means of Ni-NTA agarose (Qiagen) using $8 \mathrm{M}$ urea as described by the manufacturer. The resulting protein was dialysed against multiple changes of phosphate-buffered saline and subsequently used for immunization. NLRX1 antigen was injected into mice and monoclonal antibodies were produced by standard methodology.

Real-time PCR and reverse transcription. Complementary DNA was generated from total RNA using random priming and MMLV reverse transcriptase (Invitrogen). Real-time PCR was performed using QuantiTect SYBR Green PCR Master Mix (Qiagen) in triplicate experiments and analysed on an AB Prism 7700 analyser (Applied Biosystems). All real-time values were normalized to $18 \mathrm{~S}$ rRNA. The real-time oligonucleotides used in the study were: $18 \mathrm{~S}$ forward, 5'-GCTGCTGGCACCAGACTT, $18 \mathrm{~S}$ reverse, 5' -CGGCTACCACATCCAAGG; IFNB forward, CATTACCTGAAGGCCAAGGA, IFNB reverse, 5'-CAATTGTCCAGTCCCAGAGG; IFNA4 forward, CCTAGAGGCCGAAGTTCAAG, IFNA4 reverse, TTGTGCCAGGAGTATCAAGG; IL10 forward, ATGCCCCAAGCTGAGAACCAAGACCCA, IL10 reverse, 5'-TCTCAAGGGGCTGGGTCAGCTATCCA; IL6 forward, CGGGAACGAAAGAGAAGCTCTA, IL6 reverse, 5'-GGCGCTTGTGGAGAAGGAG.

Immunofluorescent staining, immunogold and mitochondrial localization. HeLa cells were plated on two-well glass chamber slides and transfected with $1.0 \mu \mathrm{g}$ per well GFP-NLRX1 or pcDNA3 using Fugene 6 (Roche Applied Science), or were stained with NLRX1 antibody. Anti-mouse Alexa Fluor 488 (Molecular Probes) was used to stain for endogenous NLRX1. Exogenous and endogenous NLRX1 localizations were visualized on a Zeiss LSM5 PASCAL confocal microscope. To visualize co-localization of NLRX1 with the mitochondria, HeLa cells were stained with MitoTracker (Invitrogen) and endogenous NLRX1. For immunogold labelling of NLRX1, 293T cells were fixed in $2 \%$ paraformaldehyde and $0.5 \%$ glutaraldehyde. After fixation, cells were embedded in London Resin White and sectioned parallel to the substrate at $80 \mathrm{~nm}$. Sections were labelled using a 1:50 dilution of a monoclonal antibody to NLRX1, 1:100 polyclonal antibody to MAVS or TOM20. Goat anti-mouse or anti-rabbit IgG antibody conjugated to a 5 or $15 \mathrm{~nm}$ colloidal gold particle (Polysciences) was used as a secondary antibody. Immunogold labelling was followed by staining in uranyl acetate and lead citrate, and sections were photographed using a LEO EM910 transmission electron microscope (LEO Electron Microscopy Inc.) at $80 \mathrm{kV}$. To further test mitochondrial localization, cytoplasmic, nuclear, crude mitochondria and other cellular compartments were isolated from HEK293T cells using a deferential centrifugation method, and each was immunoblotted for known proteins and NLRX1. The mitochondrial fractions were partially sonicated to break the outer membrane; this was applied to a $0.4-1.8 \mathrm{M}$ sucrose gradient and fractions were taken from the top. Each fraction was immunoblotted for outer (VDAC1 and MAVS) and inner (COXIV) mitochondrial membrane fractions in addition to NLRX1. Trypsin protection assays were performed by the addition of $1.0 \mathrm{mg} \mathrm{ml}^{-1}$ trypsin for $10 \mathrm{~min}$, and fractions were lysed in RIPA for immunoblotting.

Luciferase assays. HEK293T or TLR3-HEK293T $\left(1 \times 10^{4}\right.$ cells $)$ were plated in 96-well plates and transfected $24 \mathrm{~h}$ post-transfection with $25 \mathrm{ng}$ of IFNB, activator protein-1 (AP-1), NFKB or $p 53$ luciferase reporter construct and $0.1,1,10$ or $100 \mathrm{ng}$ of HA-NLRX1 using FuGENE6 (Roche Applied Science). Empty pcDNA3 was used to maintain equal DNA amounts between wells. Exogenous MAVS, extracellular poly(I:C) or transfected poly(I:C) were used as an agonist for each reporter except the p53 reporter, in which p53 plasmid was used as an agonist. Cells were harvested $18-24 \mathrm{~h}$ post transfection, and luciferase units were measured by standard methodology.

Immunoprecipitation and western blots. Lysates were prepared after co-transfection of HA-NLRX1 or pcDNA3 with Flag-MAVS. Cells were lysed for $45 \mathrm{~min}$ in cold RIPA buffer containing protease inhibitors (Roche). All lysates were cleared and incubated with anti-HA-agarose beads (Roche) overnight. Beads were washed four times with RIPA, and immunoprecipitates were eluted with SDS-PAGE loading buffer. SDS-PAGE was performed on lysates and immunoprecipitates transferred to nitrocellulose membrane. To detect interacting FlagMAVS, standard immunoblotting was performed using M5 anti-Flag-agarose beads (Sigma). Endogenous co-immunoprecipitations were performed as described by using either of two antibodies raised against MAVS (Abcam), followed by incubation with immobilized protein A/G ultralink (Pierce). Immunoblotting on immunoprecipitates was performed as described with anti-NLRX1. For all blots, supersignal chemiluminescence pico reagent (Pierce) was used for protein detection.

NLRX1 siRNA. Non-targeting, GAPDH or NLRX1 siRNA ON-TARGETplus SMARTpool oligonucleotides (Dharmacon) were transfected according to the commercial protocol. The sequences of each siRNA oligonucleotide pool used in this study were as follows: non-targeting, 5' -AUGAACGUGAAUUGCUCAA-3', 5'-UAAGGCUAUGAAGAGAUAC-3', 5' -AUGUAUUGGCCUGUAUUAG-3', 5'-UAGCGACUAAACACAUCAA-3'; GAPDH, 5' -CAACGGAUUUGGUCGUAUU-3', 5' -GACCUCAACUACAUGGUUU-3', 5'-UGGUUUACAUGUUCCAAUA-3', 5'-GUCAACGGAUUUGGUCGUA-3'; and NOD9, 5'-AUCCCGACGGAAGAUGUGCUU-3', 5'-UUGCGCAGCAGGUUGACGAUU-3', 5'-UCACGUGAGCUGGUGUGGUU-3', 5' -UCUUGCGCAAACCCAGCACUU-3'. HEK293T $\left(2 \times 10^{5}\right.$ cells $)$ were plated in 6-well plates in antibiotic-free DMEM-H. At 50\% confluence, $100 \mathrm{nM}$ siRNA was transfected using the siRNA optimized transfection reagent Dharmafect I (Dharmacon). To determine efficiency of protein knockdown, at $72 \mathrm{~h}$ post-transfection, cells were lysed in RIPA for immunoblot with NLRX1 and GAPDH antibodies. Small interfering RNA cells were transfected with MAVS plasmid or infected with Sendai virus at $72 \mathrm{~h}$, and cells were harvested for real-time PCR the next day. Dimerization of IRF3 was detected using sc-9082 antibody (Santa Cruz, Inc.) and after the indicated treatments. Native gel electrophoresis was performed using the NativePAGE Novex BIS-Tris Mini Gel System and corresponding protocol (Invitrogen, Inc.). Viral infections. HEK293T cells were plated in 96-well plates at a density of $1 \times 10^{4}$ cells and incubated overnight. Viral infection was performed when $60 \%$ cell confluence was reached. Culture media was replaced by serum-free DMEM, and Sendai virus (Cantell strain; Charles River, SPAFAS) or GFP-Sindbis virus was added into the media at concentration of 80 haemagglutinating units per $\mathrm{ml}$ and 5 multiplicity of infection (MOI), respectively. After $1 \mathrm{~h}$ incubation, extracellular virus was removed by washing cells two times with serum-containing media. Cells and supernatant were harvested $18 \mathrm{~h}$ post infection for real-time PCR analysis and enzyme-linked immunosorbent assay (ELISA). IFN- $\beta$ protein was quantified by ELISAs (PBL Technologies). GFP-labelled Sindbis virus was detected by means of flow cytometry in the FL-1 channel on a four-degree logarithmic scale, and the percentage GFP-positive cells were calculated. GFP analysis was gated from live cells on the basis of size parameters, forward scatter versus side scatter.

23. Su, A. I. et al. Large-scale analysis of the human and mouse transcriptomes. Proc. Natl Acad. Sci. USA 99, 4465-4470 (2002).

24. Nakai, K. \& Horton, P. PSORT: a program for detecting sorting signals in proteins and predicting their subcellular localization. Trends Biochem. Sci. 24, 34-36 (1999). 
25. Duncan, J. A. et al. Cryopyrin/NALP3 binds ATP/dATP, is an ATPase, and requires ATP binding to mediate inflammatory signaling. Proc. Natl Acad. Sci. USA 104, 8041-8046 (2007). 